\title{
Gendermedizin: Patientinnen unterscheiden sich von Patienten
}

\author{
Nicole Steck ${ }^{a}, b$, Lisa Marxt ${ }^{c}$, Daniel Candinas ${ }^{a}$, Beatrice Beck Schimmer ${ }^{d}$, Catherine Gebhard ${ }^{e, f}$

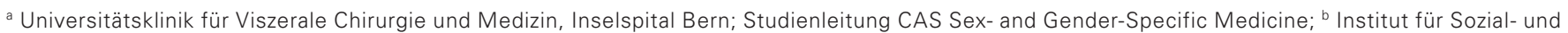 \\ Präventivmedizin, Universität Bern; ${ }^{c}$ Geschäftsstelle Direktorium Universitäre Medizin Zürich, Universität Zürich; ${ }^{d}$ Direktorin Universitäre Medizin Zürich, \\ Universität Zürich; ${ }^{e}$ Klinik für Nuklearmedizin, UniversitätsSpital Zürich; Vorsitz Programmleitung CAS Sex-and Gender-Specific Medicine; \\ ${ }^{\dagger}$ Center for Molecular Cardiology, Universität Zürich
}

\begin{abstract}
Ein Universalkonzept ist in der Erforschung und Behandlung von Krankheiten längst nicht mehr sinnvoll. Das belegt eine wachsende Anzahl von Studien. Während im Zeitalter der personalisierten Präzisionsmedizin bereits Therapiekonzepte auf einzelne Gene abgestimmt werden, wird der Unterschied zwischen Mann und Frau in der Fachliteratur und in der klinischen Routine aber kaum berücksichtigt.
\end{abstract}

\begin{abstract}
Die Gendermedizin sorgt für Schlagzeilen: So sind Herzerkrankungen bei Frauen häufiges Thema in den Medien [1]. Gemäss einer kürzlich veröffentlichten Studie des Zürcher Stadtspitals Triemli zögern Frauen bei einem Herzinfarkt länger als Männer, bis sie medizinische Hilfe in Anspruch nehmen [2]. Ein Grund für diesen fatalen Zeitverlust ist, dass bei Frauen häufig andere Symptome auftreten als bei Männern und der Herzinfarkt immer noch als typische Männerkrankheit gesehen wird. Dies erschwert die richtige Einschätzung und die rasche Einweisung an die zuständigen Notfalleinrichtungen [3]. Als weiteres Beispiel sei das Schlafmittel Zolpidem genannt. Zolpidem sorgte für medialen Wirbel, nachdem sich Berichte über morgendliche Autounfälle von Frauen nach der Einnahme von Zolpidem am Vorabend häuften $[4,5]$. Deshalb empfiehlt heute ein Warnhinweis eine niedrigere Dosierung für Frauen.
\end{abstract}

\section{Universitäten Bern und Zürich bieten neu Weiterbildung \\ zu geschlechtsspezifischer Medizin an}

Die Universitäten Bern und Zürich bieten neu einen gemeinsamen CAS-Weiterbildungsstu diengang zu geschlechtsspezifischer Medizin an. Das Ziel ist es, Schweizer Ärztinnen und Ärzte bezüglich der geschlechtsspezifischen Medizin zu sensibilisieren, die individuelle Behandlung von Patientinnen und Patienten zu verbessern und die entsprechende Forschung voranzutreiben. In insgesamt elf Modulen werden ab Mai 2020 geschlechtsspezifische Aspekte in den unterschiedlichen medizinischen Fachrichtungen sowie in der medizinischen Forschung aufgezeigt und diskutiert. Die Referierenden - Expertinnen und Experten aus dem In- und Ausland - werden den Teilnehmenden die neuste Evidenz in den verschiedenen Bereichen aufzeigen, aber auch allfällige Forschungslücken und ihre Konsequenzen im klinischen Alltag thematisieren.
Diese Beispiele sind keine Einzelfälle. Das beweist ein Blick in die medizinischen Fachpublikationen der letzten Jahre. Jährlich werden 8000 bis 9000 Artikel zum Thema Sex und Gender in der Medizin publiziert. Ein Artikel im Fachmagazin Nature sorgte im März 2019 für Aufsehen. Dieser fasste die neueste Evidenz zusammen und verdeutlichte, dass bei weiblichen und männlichen Mäusen unterschiedliche Mechanismen zurSchmerzentstehung beitragen und Schmerzmittel entsprechend unterschiedlich wirksam sind [6]. Ein kürzlich in der Zeitschrift Lancet veröffentlichter Artikel wies darauf hin, dass Frauen mit Herzinsuffizienz von niedrigeren Dosierungen der herzwirksamen Medikamente profitieren würden [7]. Andere kürzlich publizierte Arbeiten berichteten über die geschlechtsspezifische Funktionsweise der Nieren [8] oder Geschlechterunterschiede bei der Tumorbiologie und der Wirkung von Chemotherapeutika [9].

\section{Wie und warum sich Männer und Frauen unterscheiden}

Vielfältig sind die Gründe für die Unterschiede zwischen Männern und Frauen bei Prävalenz, Manifestation und Verlauf von Krankheiten. Eine wichtige Rolle spielen die Geschlechtshormone und ihr Einfluss auf verschiedene Vorgänge im menschlichen Körper. Zudem haben Frauen und Männer zwar ein praktisch identisches Genom, ein Grossteil der Gene wird aber unterschiedlich exprimiert [10]. Körpergrösse und Gewicht, Muskelmasse, Körperfett und Wasseranteil sind ebenfalls geschlechtsabhängig. 


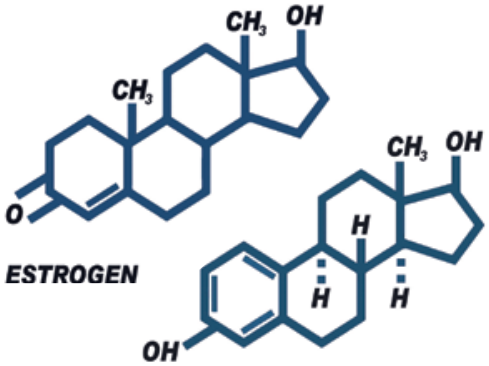

TESTOSTERONE
Neben den biologischen Unterschieden spielen auch kulturell und sozial bedingte Verhaltensweisen, die sogenannte «Gender»Dimension, eine grosse Rolle. Frauen und Männer nehmen Symptome anders wahr, verarbeiten und kommunizieren diese anders. Wie wichtig das soziale Geschlecht bei Herzerkrankungen ist, wurde kürzlich in der Zeitschrift JACC publiziert [11]. Rund 900 Studienteilnehmende beantworteten einen Fragebogen, der Variablen erfasste, die mit dem Geschlecht assoziiert werden wie z.B. Kinderbetreuung, soziale Unterstützung, Bildungsstand oder Einkommen. Gestützt auf die Antworten konnte ein sogenannter Genderindex von sehr maskulin bis sehr feminin abgeleitet werden. Die Studie zeigte, dass feminine Eigenschaften - unabhängig vom biologischen Geschlecht - mit einer deutlich höheren Sterblichkeit nach Herzinfarkt

\section{Eine wichtige Rolle spielen die Geschlechts- hormone und ihr Einfluss auf verschiedene Vorgänge im menschlichen Körper.}

assoziiert waren als maskuline Eigenschaften. Soziokulturell geprägte Verhaltensweisen spielen auch eine Rolle bei der Interaktion von Ärzten bzw. Ärztinnen und Patienten bzw. Patientinnen. So haben Forschende aus Yale aufgezeigt, dass Ärztinnen und Ärzte Mädchen als schmerzempfindlicher einschätzen als Jungen [12].

\section{Prototyp Mann}

Auch gilt in der Medizin nach wie vor der Mann als Prototyp. Die Manifestation und Symptomatik von Krankheiten beim männlichen Patienten gelten als

\section{Das Wichtigste in Kürze}

- Männer und Frauen unterscheiden sich bei Prävalenz, Manifestation und Verlauf von Krankheiten.

- Dennoch wird die Bedeutung des Geschlechts immer noch in vielen medizinischen Studien ignoriert, Frauen sind als Teilnehmende seit dem Contergan-Skandal deutlich unterrepräsentiert.

- In der Grundlagenforschung werden Daten zu 90\% an männlichen Tieren erhoben, nur 5\% der Forschungsarbeiten werden an weiblichen Zellen durchgeführt.

- Aber der Geschlechts-Bias liegt nicht immer auf Seiten der Frauen: Die Osteoporose beim Mann ist eines der am meisten vernachlässigten Krankheitsbilder Europas.

- Die Universitäten Bern und Zürich bieten neu einen gemeinsamen CAS-Weiterbildungsstudiengang zu geschlechtsspezifischer Medizin an (mehr dazu im Kasten). «typisch», während Symptome, die öfters bei Frauen beobachtet werden, oftmals als «atypisch» bezeichnet werden. Neue Medikamente werden vorwiegend an Männern getestet, obwohl geschlechtsspezifische Unterschiede für die Verteilung und Metabolisierung von Arzneistoffen eindeutig nachgewiesen sind. Die Unterrepräsentation von Frauen in Arzneimittelstudien hat unter anderem historische Gründe: Nach dem Contergan-Skandal wurde in den 1960er Jahren möglichst darauf verzichtet, Frauen in klinische Versuche einzuschliessen. Mit diesem Vorgehen sollten mögliche Schäden an ungeborenem Leben verhindert werden. Erst in den späten 1990er Jahren setzte sich die Erkenntnis durch, dass sich Wirkung und Nebenwirkungen von Arzneimitteln bei Männern und Frauen unterscheiden können [13]. Leitlinien wurden seither angepasst, und Behörden, Förderungsinstitutionen sowie wissenschaftliche Zeitschriften sind Aufrufen gefolgt, Regelungen zur geschlechtsspezifischen Forschung und Medizin zu implementieren.

Trotz dieser Massnahmen wird die Bedeutung des Geschlechts immer noch in vielen medizinischen Studien ignoriert. Frauen sind in Arzneimittelstudien nach wie vor deutlich unterrepräsentiert, wie eine FDA-Analyse aus dem Jahr 2018 verdeutlicht [14]. Auch in der Grundlagenforschung wird die Frage nach dem Geschlecht kaum gestellt: Daten werden zu 90\% an männlichen Tieren erhoben, und nur 5\% der Forschungsarbeiten werden an weiblichen Zellen durchgeführt [15-18]. Eine Angabe zum Geschlecht der verwendeten Tiere und Zellen ist meistens nicht vorhanden. Damit gehen bereits in diesem frühen Stadium der Forschung wichtige Erkenntnisse verloren. Bedenklich ist, dass der Geschlechts-Bias zulasten der weiblichen Tiere in den letzten Jahren zugenommen hat [19]. Die Gründe hierfür sind unklar.

\section{L'essentiel en bref}

- La prévalence, la manifestation et l'évolution des maladies diffèrent selon le sexe.

- Pourtant, aujourd'hui encore, de nombreuses études médicales ne tiennent pas compte de l'importance du sexe. Depuis le scandale du thalidomide, les femmes sont clairement sous-représentées parmi les participants.

- $\quad$ Dans la recherche fondamentale, $90 \%$ des données sont collectées sur des animaux mâles et seulement $5 \%$ des recherches sont effectuées sur des cellules femelles.

- Mais la recherche n'est pas toujours biaisée au détriment des femmes: I'ostéoporose masculine est l'un des tableaux cliniques les plus négligés en Europe.

- Les universités de Berne et de Zurich proposent désormais un cycle de formation post-graduée commun, sanctionné par un CAS, en médecine différenciée selon le sexe (informations complémentaires dans l'encadré). 
Eine Erklärung für den männlichen Fokus in der Medizin liegt darin, dass die Medizin lange von Männern dominiert wurde. Männer erforschten Krankheiten, publizierten die Resultate, erstellten die Leitlinien und behandelten die Patienten - und Patientinnen. Die Folge ist häufig eine spezifisch männliche Betrachtungsweise von Krankheitsbildern und Therapieverlauf.

\section{Auch Männer erkranken an Osteoporose}

Aber der Geschlechts-Bias liegt nicht immer auf Seiten der Frauen: So ist die Osteoporose beim Mann eines der am meisten vernachlässigten Krankheitsbilder Europas. Da Osteoporose als typische Frauenkrankheit gesehen wird und Diagnostik und Therapie auf eine weibliche Population abgestimmt sind, wird die Erkrankung bei Männern unterdiagnostiziert und/oder nicht adäquat behandelt. Dies steht im deutlichen Gegensatz zu dem erheblichen Frakturrisiko, das bei Männern infolge des Knochenschwundes auftritt [20]. So klar die Forderung nach einer geschlechtsbezogenen Forschung und Patientenbehandlung ist, so schleppend ist ihre Umsetzung. Dabei läge im besseren Verständnis geschlechtsspezifischer Mechanismen eine Chance für innovative Behandlungsmethoden. Beispielsweise hat die geschlechtsspezifische Analyse von Hormon-regulierten Vorgängen bei Tumorerkrankungen zu verbesserten Therapieansätzen bei Immuntherapien beigetragen [21]. Dennoch gibt es aktuell nur zwei Zentren in Europa, die sich diesem Gebiet widmen. In der Lehre wird der Faktor Geschlecht ebenfalls kaum wahrgenommen: Die Integration von geschlechtsspezifischer Medizin in die medizinischen Curricula ist in der Schweiz und in Europa nicht geregelt, und es fehlen übergreifende Konzepte zur besseren inhaltlichen und strukturellen Verankerung dieser Thematik. Der Bundesrat ist sich der Problematik bewusst. «Es gibt Hinweise darauf, dass den geschlechtsspezifischen Unterschieden in der Medizin nicht ausreichend Rechnung getragen wird», schreibt er in der Antwort auf entsprechende Vorstösse aus dem Nationalrat.

Die Gendermedizin, eine noch junge Disziplin, soll nun die bestehenden Wissenslücken in Forschung, Lehre und klinischer Medizin schliessen. Es ist offensichtlich, dass eine Effektivitätssteigerung im Gesundheitssystem nur dann erzielt werden kann, wenn gezielt auf die Bedürfnisse des einzelnen Menschen eingegangen wird. Ein erster Schritt in Richtung dieser individualisierten Medizin ist die Berücksichtigung von Geschlechtsunterschieden.

Bildnachweis

(c) Monikabaumbach | Dreamstime.com
Literatur

1 Zeit Online, Dpa, AFP cck. Deutscher Herzbericht: Das schwache Herz der Frauen. Zeit Online. 2018. https://www.zeit.de/wissen/ gesundheit/2018-01/deutscher-herzbericht-erkrankungen-herzinfarkt-risiko-therapie

2 Meyer MR, Bernheim AM, Kurz DJ, et al. Gender differences in patient and system delay for primary percutaneous coronary intervention: current trends in a Swiss ST-segment elevation myocardial infarction population. Eur Hear J Acute Cardiovasc Care. 2019;8:283-90. doi:10.1177/2048872618810410

3 Clerc Liaudat C, Vaucher P, De Francesco T, et al. Sex/gender bias in the management of chest pain in ambulatory care. Womens Health (Lond Engl). 2018; 14: 1-9 . 2018;14:1745506518805641. doi:10.1177/1745506518805641

4 Greenblatt DJ, Harmatz JS, Singh NN, et al. Gender differences in pharmacokinetics and pharmacodynamics of zolpidem following sublingual administration. J Clin Pharmacol. 2014;54:282-90. doi:10.1002/jcph.220

5 FDA. Risk of next morning impairment after use of insomnia drugs; FDA requires lower recommended doses for certain drugs containing zolpidem (Ambien, Ambien CR, Edluar, and Zolpimis) 2013. https://www.fda.gov/media/84992/download

6 Dance A. Why the sexes don»t feel pain the same way. Nature. 2019;567:448-50. doi:10.1038/d41586-019-00895-3

7 Santema BT, Ouwerkerk W, Tromp J, et al. Identifying optimal doses of heart failure medications in men compared with women: a prospective, observational, cohort study. Lancet. 2019;394:125463. doi:10.1016/S0140-6736(19)31792-1

$8 \mathrm{Hu}$ R, McDonough AA, Layton AT. Functional implications of the differences in transporters» abundance along the rat nephron: modeling and analysis. Am J Physiol Physiol. 317: F1462-F1474, 2019. doi:10.1152/ajprenal.00352.2019

9 Wagner AD, Oertelt-Prigione S, Adjei A, et al. Gender medicine and oncology: report and consensus of an ESMO workshop. Ann Oncol Published Online First: 15 October 2019. doi:10.1093/annonc/ mdz414

10 Gershoni M, Pietrokovski S. The landscape of sex-differential transcriptome and its consequent selection in human adults. BMC Biol. 2017;15:7. doi:10.1186/s12915-017-0352-z

11 Pelletier R, Khan NA, Cox J, et al. Sex Versus Gender-Related Characteristics. J Am Coll Cardiol. 2016;67:127-35. doi:10.1016/j. jacc.2015.10.067

12 Earp BD, Monrad JT, LaFrance M, et al. Featured Article: Gender Bias in Pediatric Pain Assessment. J Pediatr Psychol. 2019;44:40314. doi:10.1093/jpepsy/jsy104

13 Martin RM, Biswas PN, Freemantle SN, et al. Age and sex distribution of suspected adverse drug reactions to newly marketed drugs in general practice in England: analysis of 48 cohort studies. $\mathrm{Br} \mathrm{J}$ Clin Pharmacol. 1998;46:505-11. doi:10.1046/j.1365-2125.1998.00817.x

14 Scott PE, Unger EF, Jenkins MR, et al. Participation of Women in Clinical Trials Supporting FDA Approval of Cardiovascular Drugs. J Am Coll Cardiol. 2018;71:1960-9. doi:10.1016/j.jacc.2018.02.070

15 Shah K, McCormack CE, Bradbury NA. Do you know the sex of your cells? Am J Physiol Physiol. 2014;306:C3-18. doi:10.1152/ajpcell.00281.2013

16 Taylor K, Vallejo-Giraldo C, Schaible NS, et al. Reporting of sex as a variable in cardiovascular studies using cultured cells. Biol Sex Differ. 2011;2:11. doi:10.1186/2042-6410-2-11

17 Mogil JS. Sex differences in pain and pain inhibition: multiple explanations of a controversial phenomenon. Nat Rev Neurosci. 2012;13:859-66. doi:10.1038/nrn3360

18 Zucker I, Beery AK. Males still dominate animal studies. Nature. 2010;465:690. doi:10.1038/465690a

19 Ramirez FD, Motazedian P, Jung RG, et al. Sex Bias Is Increasingly Prevalent in Preclinical Cardiovascular Research: Implications for Translational Medicine and Health Equity for Women A Systematic Assessment of Leading Cardiovascular Journals Over a 10-Year Period. Circulation. 2017;135:625-6. doi:10.1161/CIRCULATIONAHA.116.026668

20 Szulc P, Kaufman JM, Orwoll ES. Osteoporosis in Men J Osteoporos. 2012;2012:1-5. doi:10.1155/2012/675984

21 Tannenbaum C, Ellis RP, Eyssel F, et al. Sex and gender analysis improves science and engineering. Nature. 2019;575:137-46. doi:10.1038/s41586-019-1657-6 